EPJ Web of Conferences 32, 03006 (2012)

DOI: $10.1051 /$ epjconf/20123203006

(C) Owned by the authors, published by EDP Sciences, 2012

\title{
Commissioning of inline ECE system within waveguide based ECRH transmission systems on ASDEX upgrade
}

\author{
W.A. Bongers ${ }^{1}$, W. Kasparek ${ }^{2}$, N. Doelman ${ }^{3}$, R. van den Braber ${ }^{3}$, H. van den Brand ${ }^{1,4}$, F. Meo ${ }^{5}$, \\ M.R. de Baar ${ }^{1,4}$, F.J. Amerongen ${ }^{1}$, A.J.H. Donné ${ }^{1,4}$, B.S.Q. Elzendoorn ${ }^{1}$, V. Erckmann ${ }^{6}$, \\ A.P.H. Goede ${ }^{1}$, L. Giannone ${ }^{2}$, G. Grünwald ${ }^{6}$, F. Hollman ${ }^{6}$, G. Kaas ${ }^{1}$, B. Krijger ${ }^{1}$, G. Michel ${ }^{6}$,

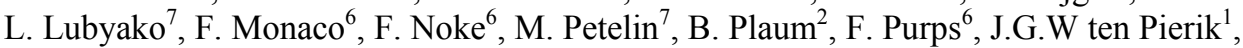 \\ C. Schüller ${ }^{1}$, J.W. Slob ${ }^{1}$, J.K. Stober ${ }^{6}$, H. Schütz ${ }^{6}$, D. Wagner ${ }^{6}$, E. Westerhof ${ }^{1}$, D.M.S. Ronden ${ }^{1}$, \\ teams at the contributing institutes ${ }^{1,2,3,5,6,7}$ and the ASDEX Upgrade Team ${ }^{6}$. \\ ${ }^{1}$ Dutch Institute For Fundamental Energy Research, NL-3439 BE Nieuwegein, The Netherlands \\ ${ }^{2}$ Institut für Plasmaforschung, Universität Stuttgart, D-70569 Stuttgart, Germany \\ ${ }^{3}$ Department of OptoMechatronics, TNO Technical Sciences, NL-2600 AD, Delft, The Netherlands \\ ${ }^{4}$ Eindhoven University of Technology, NL-5600 MB, Eindhoven, The Netherlands \\ ${ }^{5}$ Risø DTU National Laboratory for Sustainable Energy, DK-4000 Roskilde, Denmark \\ ${ }^{6}$ Max-Planck-Institut für Plasmaphysik, EURATOM Association D-17491 Greifswald, and \\ D-85748 Garching, Germany \\ ${ }^{7}$ Inst. of Applied Physics, Russian Academy of Science, 603950 Nizhny Novgorod, Russia
}

\begin{abstract}
A CW capable inline electron cyclotron emission (ECE) separation system for feedback control, featuring oversized corrugated waveguides, is commissioned on ASDEX upgrade (AUG). The system is based on a combination of a polarization independent, non-resonant, Mach-Zehnder diplexer equipped with dielectric plate beam splitters [2, 3] employed as corrugated oversized waveguide filter, and a resonant Fast Directional Switch, FADIS $[4,5,6,7]$ as ECE/ECCD separation system. This paper presents an overview of the system, the low power characterisation tests and first high power commissioning on AUG.
\end{abstract}

\section{Introduction}

For high performance tokamak plasmas, neoclassical tearing modes (NTMs) and sawteeth instabilities need to be controlled by locally applied Electron Cyclotron Current Drive (ECCD). Measuring electron temperature using Electron Cyclotron Emission (ECE) along the line of sight of ECCD offers the advantage that temperature fluctuations, resulting from NTMs and sawteeth, are measured relative to the deposition position. Such a system, in which ECE is measured in the EC transmission line, provides signals for feedback control without making any assumptions on the plasma equilibrium. These signals are insensitive to drifts in the absolute measurement or deposition position, because ECE and ECCD have similar beam propagation and are affected equally by the launcher. An inline ECE system was demonstrated to be effective on TEXTOR in a quasi-optical configuration [1]. 
For ASDEX Upgrade (AUG) a high power configuration based on oversized corrugated waveguides is required. In this paper, the design requirements for the inline ECE system on AUG are presented, resulting in a total system configuration specification (chapter 2). The low power measurements on individual parts, are described in chapter 3. Installation and Line-of-sight commissioning tests of the system on AUG are presented in chapter 4. Chapter 5 concludes with the commissioning results of the Line-of-sight system together with suggestions for further improvement.

\section{Requirements inline ECE AUG}

Unlike the quasi-optical TEXTOR system, AUG employs non-evacuated corrugated $\mathrm{HE}_{11}$ waveguides (of $87 \mathrm{~mm}$ inner diameter) to transport the mm-wave beam from the gyrotron sources to the plasma [8]. A special feature of the AUG ECCD system is the fast directional switch (FADIS) Mk II, a circular corrugated waveguide compatible version of FADIS designed by the IPF Stuttgart [4]. The main purpose of FADIS is to improve the efficiency of ECCD stabilization of NTMs by enabling the switching of the gyrotron power between two launchers positioned at the same toroidal location but at different vertical positions, such that each launcher is targeting a different phase of the NTM island. FADIS experiments performed on AUG are described in a separate paper [6]. Since FADIS is installed on AUG it can also be employed as a frequency selective diplexer, placed in lineof-sight ECE setup, to serve the purpose of separating the low-power ECE signal from the highpower ECCD, see Fig. 1.
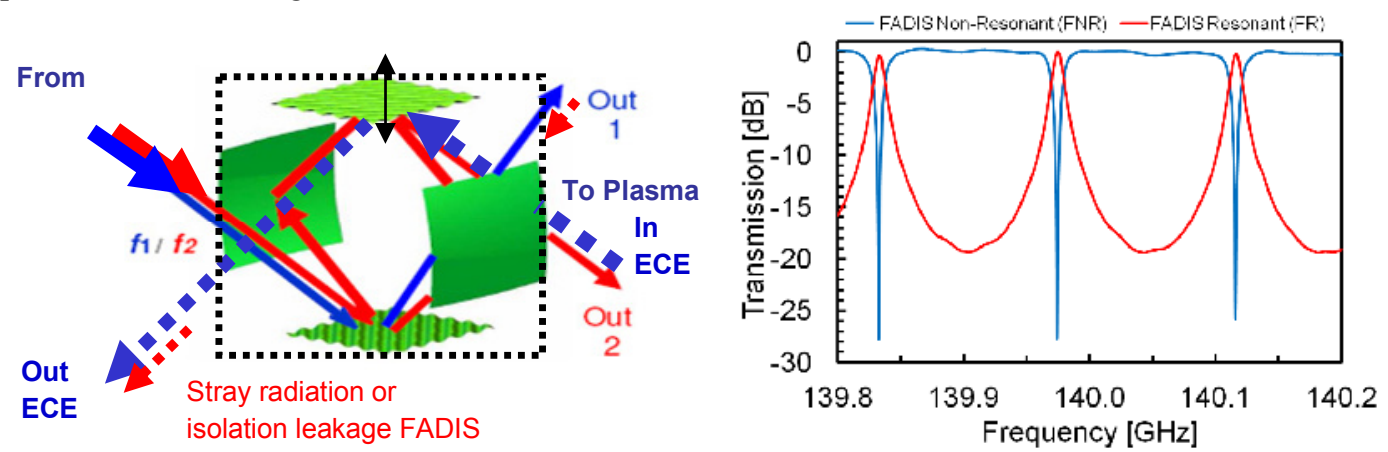

Fig. 1. Principle of FADIS for high power mm-wave application. The schematic on the left shows a 4mirror quasi-optical cavity with grating couplers for switching the high power gyrotron beam (top left) to either Out 2 (lower right) or Out 1 (upper right) ports by shifting the gyrotron frequency from $\mathrm{f} 1$ to $\mathrm{f} 2$. If the FADIS control system [7] tunes the resonant mode to the gyrotron frequency f2, by moving its upper corrugated mirror, the system serves to isolate the low power ECE radiation entering at the lower right plasma port Out 2 via the non-resonant channel coupled to the diagnostics port at the lower left (out ECE). Stray radiation or FADIS isolation leakage generated by e.g. cross coupling between the launchers, can also enter the diagnostic output of FADIS as indicated. A low power mm-wave measurement (right) shows FADIS resonant and non-resonant transmission characteristics.

As derived in [2], a $20 \mathrm{GHz}$ ECE range centred around the ECCD frequency is required at AUG. Additional requirements on the separation system of the ECE include accommodation of a possible frequency change of the gyrotrons $\sim 250 \mathrm{MHz}$ [8], whilst providing sufficient attenuation $\sim 50 \mathrm{~dB}$ in the oversized part of the diplexers in order to prevent breakdown of the standard waveguide notch filter protecting the ECE detection system. Breakdown in the notch filter could result in unsuppressed transmission of gyrotron stray radiation and damage to the notch filter and or mixers of the radiometer. From the isolation characteristics of FADIS [10] the additional filter requirements can be determined, see Fig. 2 (left). The worst case situation for inline-ECE occurs when the error in FADIS tuning is such that power is divided in equal parts over the outputs. In this case, combined with mono-mode (plane reflector) HE11 reflection somewhere in the transmission line or plasma, FADIS will suppress the reflected power fed back to the non resonant ECE output port by $6 \mathrm{~dB}$ (splitting the high power signal twice). This is very unlikely to happen. However, multi-mode 
(scattered) reflection of plasma will occur in arc or plasma resulting in $35 \mathrm{~dB}$ suppression. This requires more oversized filtering after FADIS to reach minimal $50 \mathrm{~dB}$ suppression in order to agree with the power handling capability of the standard waveguide notch filter(s) in front of the radiometer. Moreover, a pin switch would block the input of the radiometer at the start of the gyrotron where it will be in the wrong mode for a fraction of a ms and produce medium power at frequencies deviating more than $250 \mathrm{MHz}$ from the central frequency. The combined attenuation of gyrotron frequency output required for good operation of the radiometer is more than $100 \mathrm{~dB}$ (from 1 MW to $0.1 \mathrm{~mW}$ ). The setup shown in Fig. 2 (right) fulfils these requirements.
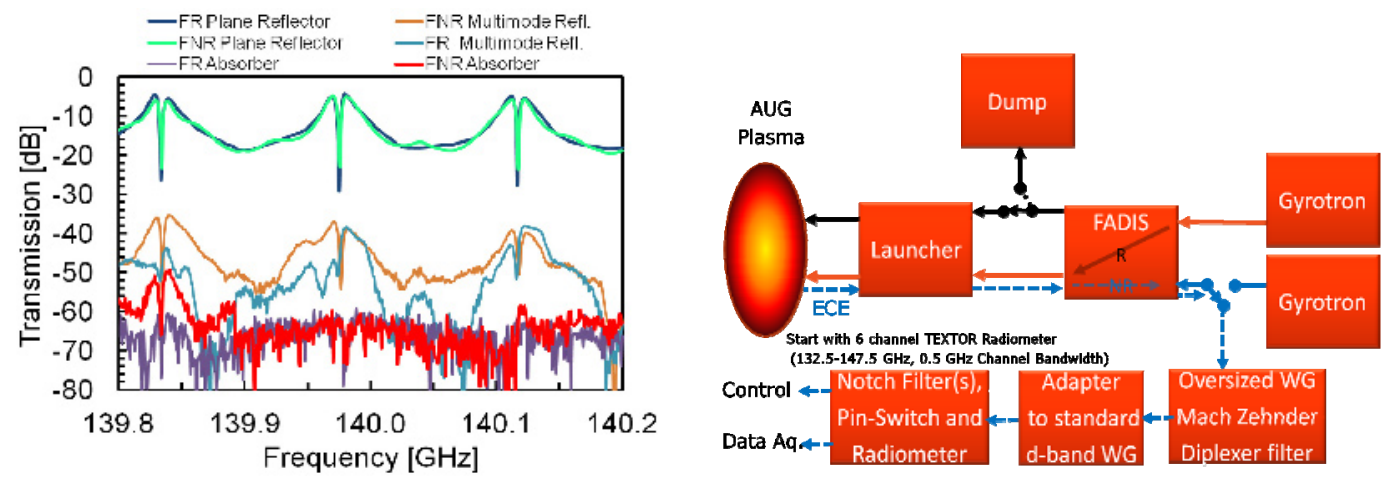

Fig. 2. Low power isolation measured on ECE output port of FADIS (left). HE 11 mode at input and at output absorber, plane reflector and multimode reflector placed at the resonant- (FR) or at the non-resonant (NFR) output [10]. The system setup of the in-line system is shown on the right: just after FADIS an oversized waveguide Mach Zehnder Diplexer filter is placed followed by an adapter to D-band standard waveguide, notch filters, pin switch and the radiometer. For correct inline operation FADIS must be tuned to the gyrotron frequency, otherwise the power will go to either the upper position or the dump depending on the position of the switch. If the in-line system is operated with the switch to the launcher, as was the case during commissioning, then FADIS non resonant frequency bandwidth ECE is measured at lower launcher and for a small bandwidth around the resonant frequencies at the upper launcher. Enabling the switch to the dump disables the upper launcher ECE.

\section{Low Power measurements}

By employing a second oversized interference filter in series with FADIS the power handling capability can be adapted to the standard waveguide notch filter(s) before the radiometer. However, in order to prevent interference and isolation decrease caused by back reflections of these filters, absorptive filters are the preferred solution. A good compact filter is a non resonant two-beam MachZehnder-type interferometer diplexer filter (MZD) [2]. Its characteristics are shown in Fig.3.

Around the gyrotron frequency, the suppression notch width and depth of the MZD is determined by the window thickness inside the beam splitters only and is independent of the phase lag path. The reason for this is that a maximum transmission is obtained at resonant frequencies while the phase lag path is switched off. Outside this frequency interval the beam splitters are operational and beating occurs between the frequency response of the interferometer and the window. The MZD is designed to provide notches at both 105 and $140 \mathrm{GHz}$. The output port (3) of the MZD is connected via an adapter, (from $87 \mathrm{~mm}$ corrugated waveguide to standard D-band waveguide), to a waveguide switch (for calibration) followed by the Bragg reflector notch filter (BRNF) described in [9]. In Fig. 4 (left) low power BRNF transmission measurements are shown and (right) the total inline ECE system filter curve is given to verify if the requirements are met just after the BRNF. As more filtering was needed in the D-band waveguide section, temporarily an additional standard waveguide notch filter (WNF) was cascaded after the BRNF. 

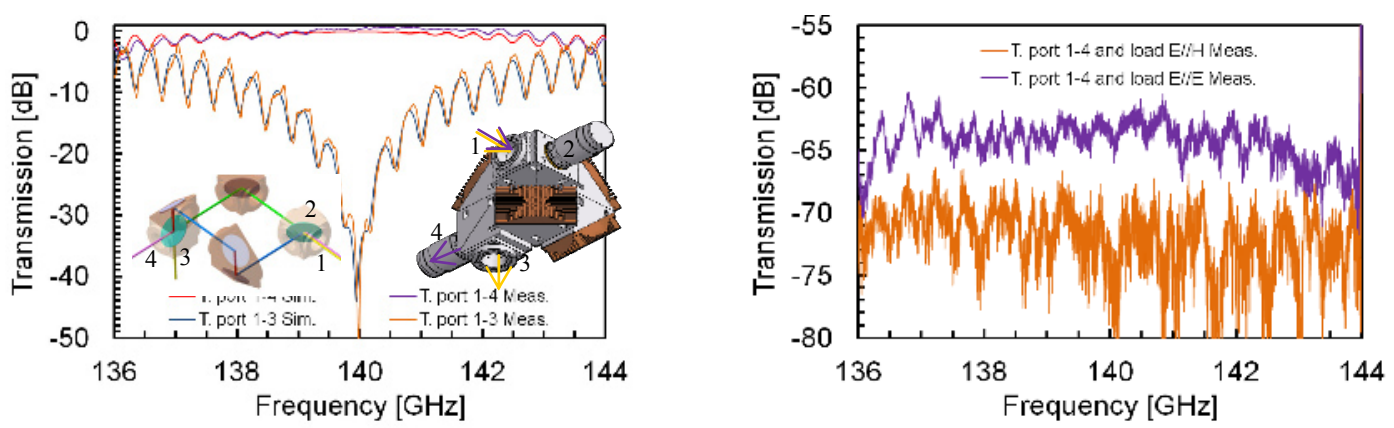

Fig. 3. Low power measurements and simulations of the transmission curve of the polarization independent MZD build from a $87 \mathrm{~mm}$ corrugated oversized waveguide (left). The input flange (1), the terminators $(2,3)$ and the cooled mitre bends are shown. Route 1-3 (yellow curve) is used as filter for ECE, which offers a minimum of 30-dB suppression of the gyrotron stray radiation over a $0.5 \mathrm{GHz}$ band caused by start-up frequency droop of the gyrotron $(<1 \mathrm{~s}$ time). The MZD also allows measuring the amount of leaked gyrotron power inside port 1 by connecting a D-band detector (preceded with $10 \mathrm{~dB}$ attenuator) on the load on port 4. The measured attenuation (calibration) of this load is given at the right for 2 polarizations.
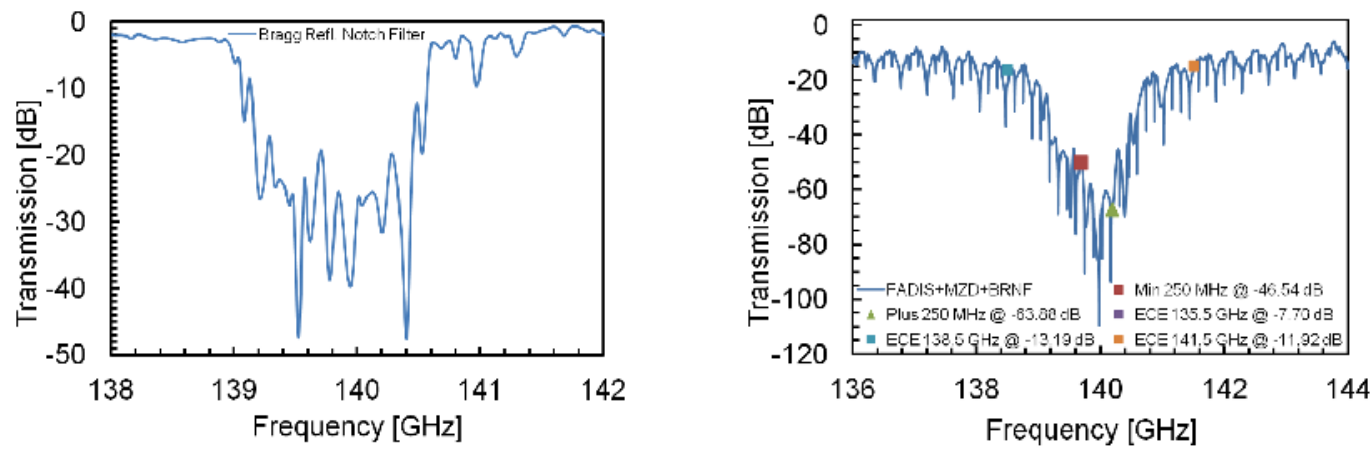

Fig. 4. Low power measurements on the prototype BRNF (left). The notch depth was designed to provide $70 \mathrm{~dB}$ attenuation [9]. However, only around $30 \mathrm{~dB}$ attenuation was reached due to mechanical tolerances not met. A new prototype is currently under construction. On the right, the calculated total ECE in-line filter curve before the radiometer is shown. The $250 \mathrm{MHz}$ frequency points and some frequencies of the surrounding ECE channels are shown. About $40 \mathrm{~dB}$ more attenuation is required to meet the specifications.
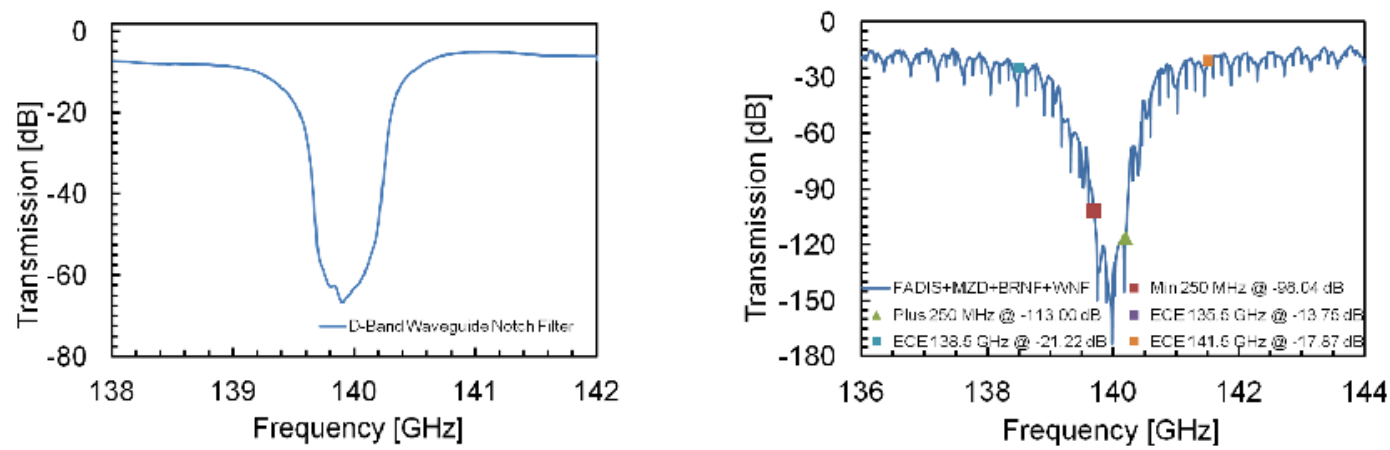

Fig. 5. Low power measurements of the WNF (left) providing a notch depth of approx. $60 \mathrm{~dB}$. The WNF shows a relatively high insertion loss of about $8 \mathrm{~dB}$. The expected total ECE inline filter curve before the radiometer is shown on the right. The $250 \mathrm{MHz}$ frequency deviation points meet requirements at the expense of high overall insertion loss at the ECE pass band, especially at the $138.5 \mathrm{GHz}$ ECE channel.

The low power characteristics of this setup is shown in Fig 5. The total inline ECE filter curve calculated is shown on the right. The requirements are met at the expense of $8 \mathrm{~dB}$ higher insertion loss of all ECE channels. After the WNF a pin switch was mounted, to suppress the beginning (1.8 
$\mathrm{ms})$ and end $(1 \mathrm{~ms})$ of the gyrotron pulse feeding FADIS, followed by the 6 channel 132.5-147.5 $\mathrm{GHz}$ frequency range radiometer (adjusted at $100 \mathrm{kHz}$ video bandwidth) which was also used for the TEXTOR experiments [1].

\section{High Power measurements: commissioning of inline ECE}

The in-line ECE system was commissioned for a number of AUG discharge conditions and control operating settings of FADIS [6,7]. During these measurements several operating modes of FADIS $[6,7]$ were employed: power division over both launchers, shown Fig. 6, and in resonance mode such that both ECE and all ECRH power pass through the upper launcher, shown in Fig. 7.

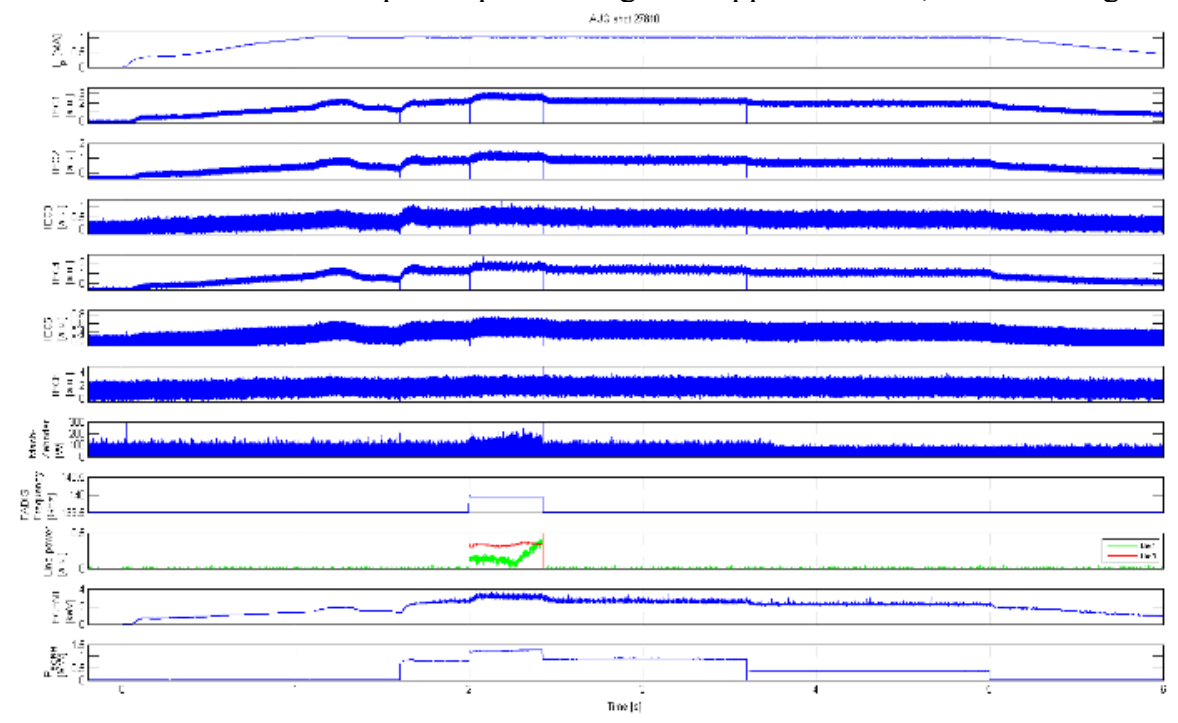

Fig. 6. Commissioning of inline ECE on AUG with a standard L-mode shot. Traces from top to bottom: plasma current, 6 inline ECE channels, leaked power in MZD, FADIS gyrotron frequency, gyrotron power at FADIS outputs, a separate ECE channel and the total power of all gyrotrons. Good correlation of inline ECE signals with separate ECE channel was found. Inline ECE channels IEC3, IEC5 and IEC6 (at 138.5, 144.5 and $147.5 \mathrm{GHz}$, respectively) show a lower signal to noise ratio caused by the high insertion losses see fig.4. The power leaked from FADIS is on the order of $200 \mathrm{~W}$, which agrees well with isolation value of Fig.1(left).

Apart from plasma heating and sawteeth instabilities also NTMs and anomalous [11] scattering, possibly in combination with edge localized modes, are observed see Fig. 7.

\section{Summary}

The first successful measurements of inline ECE were performed with the system integrated into the AUG corrugated waveguide transmission system for ECCD. Low power tests of the main components of the inline ECE system, like the MZD, are in agreement with requirements. An additional notch filter was required, at the expense of reduced $\mathrm{S} / \mathrm{N}$ ratio. A new Bragg reflector notch filter, with a deeper notch and lower insertion losses, has been purchased and this is expected to improve the poor $\mathrm{S} / \mathrm{N}$ ratio (by omitting the WNF). FADIS in switching- or beam splitting mode does not disturb the inline ECE measurements (apart from that the power flow of the gyrotron is not completely along the ECE line of sight). During commissioning of the inline ECE system on AUG

Inline ECE measurements corresponded well with conventional ECE measurements. Furthermore NTMs have been observed and anomalous scattering was possibly observed, similar to scattering measurements on TEXTOR [11]. 


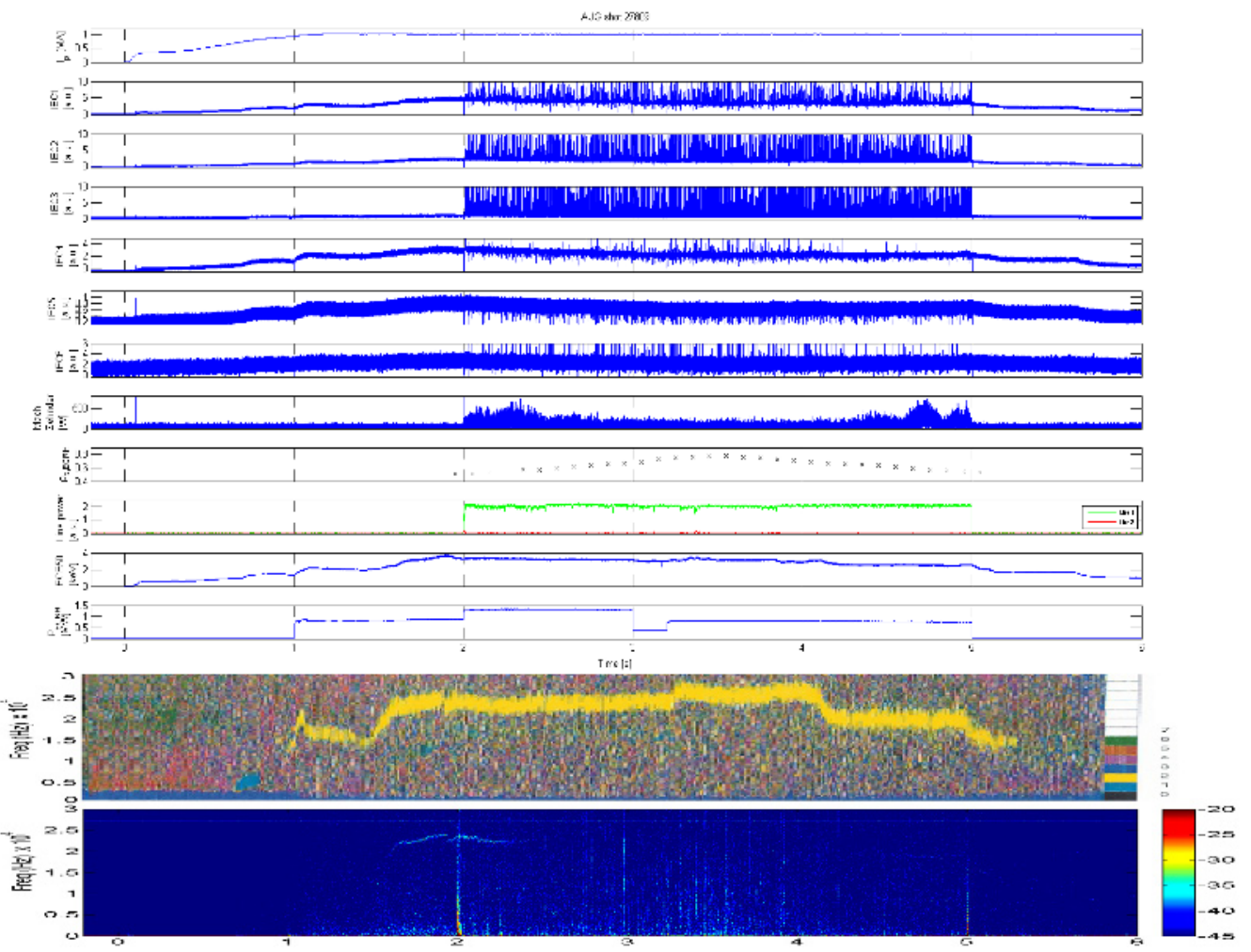

Fig. 7. AUG shot with high power NBI and ELMy H-mode Plasma. The ECCD launcher is scanned. Traces from top to bottom: plasma current, 6 inline ECE channels, leaked power in MZD, Launcher position, gyrotron power at FADIS outputs, a separate ECE channel and the total power of all gyrotrons. An FFT of ECE 4 (bottom) reveals an NTM passing by during the launcher scan, from 1.5 till $2.4 \mathrm{~s}$ this was confirmed by the FFT of the Mirnov coil signal (middle). The spikes observed on all inline ECE channels indicate scattering of ECRH (upper).

\section{References}

1. J. W. Oosterbeek, et al., Rev. Sci. Instrum. 79, (2008) 093503

2. W.A. Bongers et al., Fusion Sci. Technol. 55 (2009) 188-203

3. W.A. Bongers et al.," Utilization of collinear ECE.....”, Proc. IRMMW (2010), Rome

4. W. Kasparek et al., Fusion Sci Technol. 59 (2011) 729 - 741

5. W.A. Bongers et al.,"Progress of CW compatible....”, Proc. Strong Microwave \& THz Waves Sour. and App. (2011), Nizhny Novgorod - St. Petersburg, Russia, P. 167-168

6. W. Kasparek et al., this conference digest

7. N. Doelman, et al., this conference digest

8. D. Wagner et al., Plasma Sci., (2009), 37, 3, pp 395-402

9. D. Wagner et al., J Infrared Milli Terahz Waves (2011) 32, pp 1424-1433

10. W. Kasparek, et al., Proc. of 16th Int. Workshop EC-16, Sanya, China, 2010

11. E.Westerhof et al., Phys. Rev. Lett. 103, (2009), 12125001

This work has been performed in the framework of the NWO-RFBR Centre of Excellence (grant 047.018.002) on Fusion Physics and Technology. The work was supported by NWO, ITER-NL and the European Communities under the contract of the Association EURATOM/FOM, and was carried out within the framework of the European Fusion Programme. The views and opinions expressed herein do not necessarily reflect those of the European Commission. 